\title{
Plasmodium falciparum malaria parasite var (100smank gene expression is modified by host antibodies: longitudinal evidence from controlled infections of Kenyan adults with varying natural exposure
}

Abdirahman I. Abdi ${ }^{1,2^{*}}$, Susanne H. Hodgson ${ }^{3}$, Michelle K. Muthui ${ }^{1}$, Cheryl A. Kivisi ${ }^{1}, 2$, Gathoni Kamuyu', Domtila Kimani ${ }^{1}$, Stephen L. Hoffman ${ }^{6}$, Elizabeth Juma ${ }^{4,5}$, Bernhards Ogutu ${ }^{4,5}$, Simon J. Draper ${ }^{3}$, Faith Osier ${ }^{1}$, Philip Bejon ${ }^{1}$, Kevin Marsh ${ }^{1}$ and Peter C. Bull ${ }^{* *}$

\begin{abstract}
Background: The PfEMP1 family of Plasmodium falciparum antigens play a key role in pathogenesis of severe malaria through their insertion into the surface of parasite infected erythrocytes, and adhesion to host cells. Previous studies have suggested that parasites expressing PfEMP1 subclasses group A and DC8, associated with severe malaria, may have a growth advantage in immunologically naïve individuals. However, this idea has not been tested in longitudinal studies.

Methods: Here we assessed expression of the var genes encoding PfEMP1, in parasites sampled from volunteers with varying prior exposure to malaria, following experimental infection by sporozoites (PfSPZ). Using qPCR, we tested for associations between the expression of various var subgroups in surviving parasite populations from each volunteer and 1) the levels of participants' antibodies to infected erythrocytes before challenge infection and 2) the apparent in vivo parasite multiplication rate.
\end{abstract}

Results: We show that 1) expression of var genes encoding for group A and DC8-like PfEMP1 were associated with low levels of antibodies to infected erythrocytes (alE) before challenge, and 2) expression of a DC8-like CIDRa1.1 domain was associated with higher apparent parasite multiplication rate in a manner that was independent of levels of prior antibodies to infected erythrocytes.

Conclusions: This study provides insight into the role of antibodies to infected erythrocytes surface antigens in the development of naturally acquired immunity and may help explain why specific PfEMP1 variants may be associated with severe malaria.

Trial registration: Pan African Clinical Trial Registry: PACTR201211000433272. Date of registration: 10th October 2012.

Keywords: PfEMP1, Antibodies, P. falciparum, Immunity, Controlled human malaria infection (CHMI), Sporozoite

\footnotetext{
* Correspondence: aabdi@kemri-wellcome.org; pb642@cam.ac.uk

${ }^{1}$ KEMRI-Wellcome Trust Research Programme, CGMRC, P.O. Box 230-80108,

Kilifi County, Kenya

${ }^{7}$ Department of Pathology, University of Cambridge, 17 Tennis Court Road,

Cambridge CB2 $1 \mathrm{QP}$, UK

Full list of author information is available at the end of the article
}

(c) The Author(s). 2017 Open Access This article is distributed under the terms of the Creative Commons Attribution 4.0 International License (http://creativecommons.org/licenses/by/4.0/), which permits unrestricted use, distribution, and reproduction in any medium, provided you give appropriate credit to the original author(s) and the source, provide a link to the Creative Commons license, and indicate if changes were made. The Creative Commons Public Domain Dedication waiver (http://creativecommons.org/publicdomain/zero/1.0/) applies to the data made available in this article, unless otherwise stated. 


\section{Background}

When Plasmodium falciparum malaria parasites infect erythrocytes, they insert proteins into the erythrocyte surface that alter the properties of the infected erythrocyte surface. A large component of these inserted proteins is $P$. falciparum erythrocyte membrane protein 1 (PfEMP1) [1]. This family of parasite proteins play a key role in the pathology of severe malaria by mediating the cytoadhesion of infected erythrocytes (IE) to endothelial cells and other uninfected erythrocytes leading to IE sequestration in the microvasculature. This is thought to promote parasite survival by avoiding clearance by the spleen (reviewed in [2, 3]). Cytoadhesion is mediated by two broad categories of adhesive domains called DBL and CIDR domains, arranged in highly diverse combinations like beads on a string [4]. Because of their exposure on the surface of IE for long periods during blood-stage infection, PfEMP1 are key targets of naturally acquired immunity [5]. To evade host antibodies, $P$. falciparum switches between around 60 members of a diverse genomic repertoire of var genes, using an epigenetic mechanism that ensures only one PfEMP1 antigen is expressed at any one time by each parasite [6]. The var genes within each parasite genome expressed during childhood malaria can be broadly classified through their upstream promotor types: every parasite genome has a small number of var genes with ups $\mathrm{A}$ and ups $\mathrm{C}$ promotors, with the majority having ups $\mathrm{B}$ promotors [7].

The usefulness of PfEMP1 as vaccine targets is potentially limited by their extreme molecular diversity $[8,9]$. However, children growing up in malaria endemic areas do develop antibodies to a broad range of PfEMP1 variants and despite their molecular diversity, expression of restricted subclasses of relatively conserved PfEMP1 variants with representative var genes in every parasite genome, have been found to be associated with severe malaria [10-18]. The most important defined subsets of PfEMP1 in this regard are those with an ups A promotor, called "group A" and those containing CIDR $\alpha 1$ domains predicted to bind to endothelial protein $C$ receptor (EPCR) [19]. Though CIDR $\alpha 1$ domains have been identified within var genes with various adhesive domain architectures, they are frequently found in the context of commonly occurring combinations of cytoadhesive domains called "domain cassettes (DC)" [7]. Two examples of CIDR 1 1-containing DC reported to be associated with severe malaria [16] are DC13 (defined as: DBL $\alpha 1.7$, CIDR $\alpha 1.4$ ) and DC8 (defined as: DBL $\alpha 2$, CIDR $\alpha 1.1$, DBLß12, DBLy4/6). DC13 forms a subset of group A PfEMP1, while DC8, as strictly defined, forms a subset of var genes with ups B promotors (group B) [7]. In 3D7, the DBL $\alpha 1.7$, and CIDR $\alpha 1.4$ domains that make up DC13 are present within a single gene PF11_0521.
DC8 as strictly defined, is absent from 3D7. However, PF3D7_0600200 (PFF0010w) and PF3D7_0800300 (PF08_0140) are DC8-like in every respect apart from having CIDR $\alpha 1.8$ and CIDR $\alpha 1.6$ domains respectively in place of CIDR $\alpha 1.1$. PF3D7_0400400(PFD0020c), is DC8-like in every respect apart from having a DBL $\alpha 1.2$ domain in the place of DBL $\alpha 2$, making it a group A var.

The fitness advantages provided by these var gene subsets associated with severe malaria are not known. It is possible that adhesion to EPCR by CIDR $\alpha 1$ increases the ability of parasites to bind to diverse endothelial cells, hence lowering the rate of parasite clearance in the spleen [20]. Alternatively, because group A and DC8 PfEMP1 tend to be relatively long genes they may have more options for cytoadhesion, again allowing them an enhanced ability to avoid passage through the spleen and sustain infections [16, 21, 22]. Because these molecules tend to be more conserved, parasites expressing these variants tend to be recognised by children who have a well-developed repertoire of anti-PfEMP1 antibodies [23, 24]. Therefore, naturally acquired antibodies against these restricted subclasses of PfEMP1 variants have been proposed to confer protection against severe disease $[25,26]$.

This hypothetical trade-off between cytoadhesion and immune escape leading to the evolution of a subclass of PfEMP1 variants with a growth advantage in immunologically naïve hosts is an attractive idea; it potentially helps explain the development of immunity to severe malaria in the first few years of life despite immense overall molecular diversity in the PfEMP1 family of proteins. Several pieces of evidence indirectly support the idea. Firstly, recombinant proteins made from Group A PfEMP1 and DC8 tend to be more commonly recognized than other group B and C PfEMP1, suggesting they are antigenically more conserved [26-28]. Secondly, expression levels of group A-like PfEMP1 were associated with both severe malaria and absence of antibodies at the time of disease and young host age [10]. However, direct evidence is still needed to support 1) differential survival of this subclass of PfEMP1 variants in the face of different levels of pre-existing naturally acquired immunity and 2) an intrinsic growth advantage over other PfEMP1 types in the absence of antibody pressure, as opposed to a purely passive relation with prior exposure that is driven by their relative conservation [10, 11].

Here, using a controlled human malaria infection (CHMI) study of twenty-eight Kenyan adults infected by intramuscular administration of aseptic, purified, cryopreserved NF54 (the parent line of 3D7) P. falciparum sporozoites, (Sanaria ${ }^{\circledR}$ PfSPZ Challenge) [29], we show that pre-existing antibodies to IE $(\alpha \mathrm{IE})$ were associated with reduced group A and DC8-like var gene expression. 
One of three sets of primers (dc13, dc8-1 and dc8-2) used to amplify CIDR $\alpha 1$ domains, dc8-1, amplified a var signal associated with apparent in vivo parasite multiplication rate (PMR) after adjustment for prior levels IE antibodies.

\section{Methods \\ CHMI study design}

An open label, randomized, CHMI pilot study using PfSPZ Challenge (aseptic, purified, cryopreserved, infectious NF54 P. falciparum sporozoites (Sanaria ${ }^{\circ}$ PfSPZ [30]) was undertaken in Nairobi, Kenya [29]. Briefly, increasing doses of PfSPZ Challenge were administered intramuscularly to twenty-eight volunteers with varying degrees of prior exposure to malaria [29]. Subjects were grouped into those with minimal prior exposure to $P$. falciparum (MinExp) $(n=14)$ and those with definite prior exposure to $P$. falciparum (DefExp) $(n=14)$ determined by antibody levels to recombinant merozoite surface protein-2 (MSP-2) and whole schizont extract measured prior to CHMI [29]. The rationale for using these antigens to screen for previous exposure was based on previous published data to support their association with prior exposure [29, 31-33]. All subjects were successfully infected with malaria parasites [29]. A blood sample was collected for parasite var gene expression analysis upon blood-film positivity or at day $21(\mathrm{C}+21)$ post-CHMI in those undiagnosed by this time-point. This blood sample was processed as previously described $[10,11]$, with $100 \mu \mathrm{l}$ of RBCs, depleted of white blood cells, re-suspended in TRIzol and stored at $-80{ }^{\circ} \mathrm{C}$ until use. All subjects were treated with a curative course of atovaquone/proguanil either when blood-film positive or at $\mathrm{C}+21$.

\section{Parasite DNA extraction and 185 ribosomal RNA gene PCR} qPCR for $P$. falciparum $18 \mathrm{~S}$ ribosomal RNA (18S rRNA) gene was performed on samples collected once or twice daily as previously described [34]. These data were used to calculate each subject's PMR using simple linear regression. PMR is the fold change in number of parasites in the blood over one lifecycle $(48 \mathrm{~h})$. P. falciparum schizonts usually contain approximately 20 merozoites [35]. If these successfully invade a different red blood cell, the PMR would be 20. In CHMI studies including individuals with no naturally acquired immunity to malaria, PMR has been reported to range between 10 and $15[34,36,37]$.

\section{Parasite RNA extraction and cDNA synthesis}

Parasite RNA extraction and cDNA synthesis was performed as previously described $[10,11]$.

\section{Var transcript quantification using quantitative PCR}

Primers (Table 1) previously used to quantify expression of broad classes of var genes in qPCR [16, 38, 39] were applied as described in [40, 41]. Two housekeeping genes, Seryl tRNA synthetase and Fructose bisphosphate aldolase [16, 39] were used as reference genes for relative quantification of the expressed var genes. Primers targeting the individual var genes in 3D7 [39] were not used as the amount of parasite RNA material available for analysis was extremely limited given the low-density parasitaemia present in subjects at diagnosis. The primers used included some designed based on 3D7 genome [38] to target group A, B and C var genes (Table 1). These included two group A-targeting primers A2 and A3 renamed here as gpA3 and gpA4 (Table 1) that target the exon2 region of group A genes [38]. A third broadly specific group A primer gpA1 (originally named "Dbla1 not var3") has been shown to amplify all DBL $\alpha$ domains from group A 3D7 var genes ([16] and (Table 1).

Domain cassettes present a challenge for amplification by qPCR because they are defined as common combinations of domains whereas individual qPCR amplified domains can occur in different molecular contexts. We used primers designed to amplify DC8-like genes from field isolates [16]. Here we define DC8-like genes as those that contain individual PCR-targeted, DBL or CIDR domain sequence features present within the originally defined domain cassette 8 [7]. Specific data for amplification from the NF54-derived, 3D7 genome (Table 1) shows that dc8-1 amplifies a single group A gene PFD0020c containing a DC8-like CIDRa1.1 domain, dc8-2 amplifies two DC8-like group B genes that contain a characteristic DBL 22 domain joined to a CIDR $\alpha 1$ domain, dc8-3 and dc8-4 amplify DBL $\beta$ and DBLy domains that are found in DC8, but in 3D7 are found in both group A and group B genes (Table 1).

The Real-time quantitative PCR was carried out in duplicates in 96-well plates. The PCR reaction and cycling conditions were carried out as described in [16] using the Applied Biosystems 7500 Real-time PCR system with a cycle threshold $(\mathrm{Ct})$ set at $0.025[40,41]$. Controls with no template were included and the melt-curves analysed for non-specific amplification. The $\Delta \Delta$ ct relative quantification method was used to calculate the arbitrary transcript unit $\left(\mathrm{TU}_{\mathrm{s}}\right)$ using the formula $\left(\mathrm{TU}_{\mathrm{s}}=2^{(5-\Delta \Delta \mathrm{ct})}\right)$. Relative quantification is inappropriate for estimating proportional expression. Therefore, when calculating the proportional expression of the var subclasses, we used $\mathrm{TU}_{\mathrm{s}}$ calculated from the formula $\left(\mathrm{TU}_{\mathrm{s}}=2^{(5-\Delta \mathrm{ct})}\right)$ as described in $[40,41]$. We assigned a $\mathrm{TU}_{\mathrm{s}}$ value of zero to any reaction that did not give a detectable amplification after 40 cycles of amplification. Only samples where we could obtain amplification with the two housekeeping genes were included in the analysis. 
Table 1 The list of primers used in this study and their predicted targets

\begin{tabular}{|c|c|c|c|c|}
\hline Original primer name & Name given & Ref. & Targets in 3D7 [16] & Predicted NF54 target genes [16] \\
\hline dbla_not_var3 & gpA1 & [16] & $\begin{array}{l}\text { All group A var (DBLa1.2, DBLa1.3, DBLa1.4, } \\
\text { DBLa1.5, DBLa1.6, DBLa1.7) }\end{array}$ & $\begin{array}{l}\text { PFD0020c, PFA0015c, MAL6P1.314, } \\
\text { PFI1820w, PFD1235w, PFE1640w, } \\
\text { PF11_0008, PF08_0141, PF11_0521, } \\
\text { PF13_0003 }\end{array}$ \\
\hline A2 & gpA3 & [38] & Exon2 of group A var & \\
\hline A3 & gpA4 & [38] & Exon2 of group A var & \\
\hline cidra1.4 & dc13 & [16] & DC13 group A var (CIDRa1.4) & PF11_0521 \\
\hline cidra1.1 & dc8-1 & [16] & One group A, DC8-like var (CIDRa1.1) & PFD0020c \\
\hline dbla_cidra & $\mathrm{dc} 8-2$ & {$[16]$} & Two group B, DC8-like var (DBLa2_CIDRa1) & MAL6P1.316, PF08_0140 \\
\hline dblb12 \& dblb3/5 & dc8-3 & [16] & DC8-like var in group $A$ and $B(D B L \beta 12, D B L \beta 3)$ & $\begin{array}{l}\text { MAL6P1.316, PF08_0140, PFD0020c, } \\
\text { PF13_0003, PF11_0521 PFD1235w }\end{array}$ \\
\hline $\mathrm{dblg} 4 / 6$ & dc8-4 & [16] & DC8-like var in group $A$ and $B(D B L y 4, D B L \gamma 6)$ & PFD0020c, MAL6P1.316, PF08_0140 \\
\hline B1 & b1 & [38] & Conserved upstream of group B var & \\
\hline C1 & $\mathrm{cl}$ & [38] & Conserved upstream of group C var & \\
\hline Seryl-tRNA_synthatase & & [39] & & \\
\hline Fructose_biphosphase aldolase & & [39] & & \\
\hline
\end{tabular}

"Primer name" is the name of the primer in the original study (see reference column), "Name given" is the name given to the primer in this study. We included the primers gpA3 and gpA4 designed based on 3D7 genome (a clone of NF54) to independently capture group A expression. Primers were previously shown to amplify the 3D7 genes shown in the right-hand column [16]. Gene names in bold are group A var genes. New 3D7 gene names: PFD0020c = PF3D7_0400400; PFA0015c = PF3D7_0100300; MAL6P1.314 = PF3D7_0600400; PFI1820w = PF3D7_0937600; PFD1235W = PF3D7_0425800; PFE1640w = PF3D7_0533100; PF11_0008 = PF3D7_1100200; PF08_0141 = PF3D7_0800200; PF11_0521 = PF3D7_1150400; PF13_0003 = PF3D7_1300300;

MAL6P1.316 = PF3D7_0600200; PF08_0140 = PF3D7_0800300

\section{Infected erythrocyte surface antibodies (aIE)}

Plasma obtained from study participants before CHMI was used to assess antibodies specific to IE $(\alpha \mathrm{IE})$ with the trophozoite stages of 8 clinical isolates recently adapted into culture [42] following isolation from children living in Kilifi county, Kenya. In vitro, the NF54 parasite line predominantly expresses var2CSA [43, 44] and from our experience, this line and other long-term laboratory cultured parasite lines give poor signal when reacted with plasma from individuals with naturally acquired immunity as compared with ex vivo matured clinical isolates. For that reason, we chose recently culture-adapted Kenyan isolates [42] to increase the breadth of variant surface antigens available for recognition by naturally acquired antibodies present in the plasma of the volunteers. The 8 isolates were mixed together in equal proportion based on parasitemia to make a composite of approximately $1.5 \%$ parasitemia before reacting with the plasma from each participant. A single plasma sample from an exposed adult residing in Kilifi, Kenya was used as a positive control and four from malaria-naïve Europeans plus one-pooled European sera of $\mathrm{AB}$ blood group were used as negative controls. The assay was carried out in duplicate and in two 96-well plates, providing four data points for each participant. Reactivity of plasma against the IE was measured using flow cytometry $[10,11]$ and presented as mean of the median fluorescent intensity obtained from two plates.

\section{Anti-schizont extract and anti-MSP2 antibodies}

These data were obtained using ELISA as previously published [29].

\section{Statistical analysis}

Statistical analysis was performed using Stata version 13 and graphs were generated using GraphPad Prism version 5 .

When more than one primer was used to quantify a certain var subclass, we calculated the median transcript quantity obtained with the different primers to represent the expression of the particular var subclass as described in $[40,41]$. For example, the median of DC8 (dc8_median $)=$ the median transcript obtained with primers dc8-1, dc8-2, dc8-3, and dc8-4, and the median of group A (gpA_median) $=$ the median transcript obtained with the primers gpA1, gpA3, and gpA4. The primer to DC13, specifically designed to target CIDR $\alpha 1.4$ was not included in this global calculation because it is known to amplify only a single var gene, PF11_0521 (Table 1).

Correlation between variables was assessed using Spearman's rank correlation coefficient and Bonferroni correction was performed to adjust the $p$-value for multiple comparison (unless otherwise stated in the text, or indicted with * in the tables, quoted $p$-values are unadjusted). Linear regression analysis was used to assess whether the relationship between PMR and the transcript quantity of the var subclasses expressed by the infecting parasites was confounded by pre-existing 
antibodies to IE ( $\alpha \mathrm{IE})$. To normalize the distribution of the data before use in regression analysis, the square root of the explanatory variables was calculated except for 1) group A proportional expression (gpA_prop and gpA_prop2) that was already normally distributed and 2) the $\alpha \mathrm{IE}$ where the inverse $(1 / \alpha \mathrm{IE})$ was taken.

Participant 110 from the published study [29] was excluded from analyses that includes var gene expression data, since amplification of the reference genes was not achieved due to the low parasitaemia.

\section{Results}

Pre-existing naturally acquired alE differentially selects against group $\mathrm{A}$ and DC8-like var gene expression

To determine whether $\alpha \mathrm{IE}$ antibodies carried by each volunteer before CHMI, imposed a selection pressure on the PfEMP1 antigens expressed in parasites that escaped those antibodies and established blood infection, we tested for correlations between $\alpha \mathrm{IE}$ antibodies and expression levels of var gene subclasses encoding PfEMP1 associated with low antigenic diversity. We predicted that the parasites that establish blood-stage infection in volunteers with low naturally acquired pre-existing $\alpha \mathrm{IE}$ antibodies would express higher levels of the conserved var gene subclasses, group A and DC8-like compared to those with high pre-existing $\alpha I E$ antibodies.

The results matched well with these predictions. The transcript quantity obtained with one of the primers designed to target the majority of group A var genes [16] (gpA1) was negatively associated with $\alpha$ IE (Table 2). The associations between gpA1 and $\alpha \mathrm{IE}$ persisted after Bonferroni correction for ten comparisons (corrected $p=0.006$, Table 2). The transcript quantity of two other primers (gpA3 and gpA4) also designed to target group A var genes and used in previously published studies [38, 45] showed a trend towards a negative association with $\alpha$ IE but with borderline significance (Table 2). The median transcript quantity of group A var genes (gpA_median) as measured with the three primers gpA1, gpA3, and gpA4 designed to globally amplify group A var genes was negatively associated with aIE (Table 2). However, DC13 primers, designed to amplify a subset of group A var genes containing CIDRa1.4 domains showed no evidence for an association with $\alpha \mathrm{IE}$ antibodies.

The transcript quantity of the four primers targeting sequence features found within DC8 [16] dc8-1, dc8-2, dc8-3 and dc8-4 indicated negative trends in relation to $\alpha$ IE (Table 2). dc8-2, dc8-3, and dc8-4 transcript quantity scores were significantly negatively associated with $\alpha \mathrm{IE}$ after Bonferroni correction for ten comparisons (corrected $p$-value $=0.04,0.05$, and 0.03 for $\mathrm{dc} 8-2, \mathrm{dc} 8-3$, and $\mathrm{dc} 8-4$ respectively,
Table 2 The relationship between expression of specific var subclasses, IE surface antibodies (alE) and parasite multiplication rate (PMR)

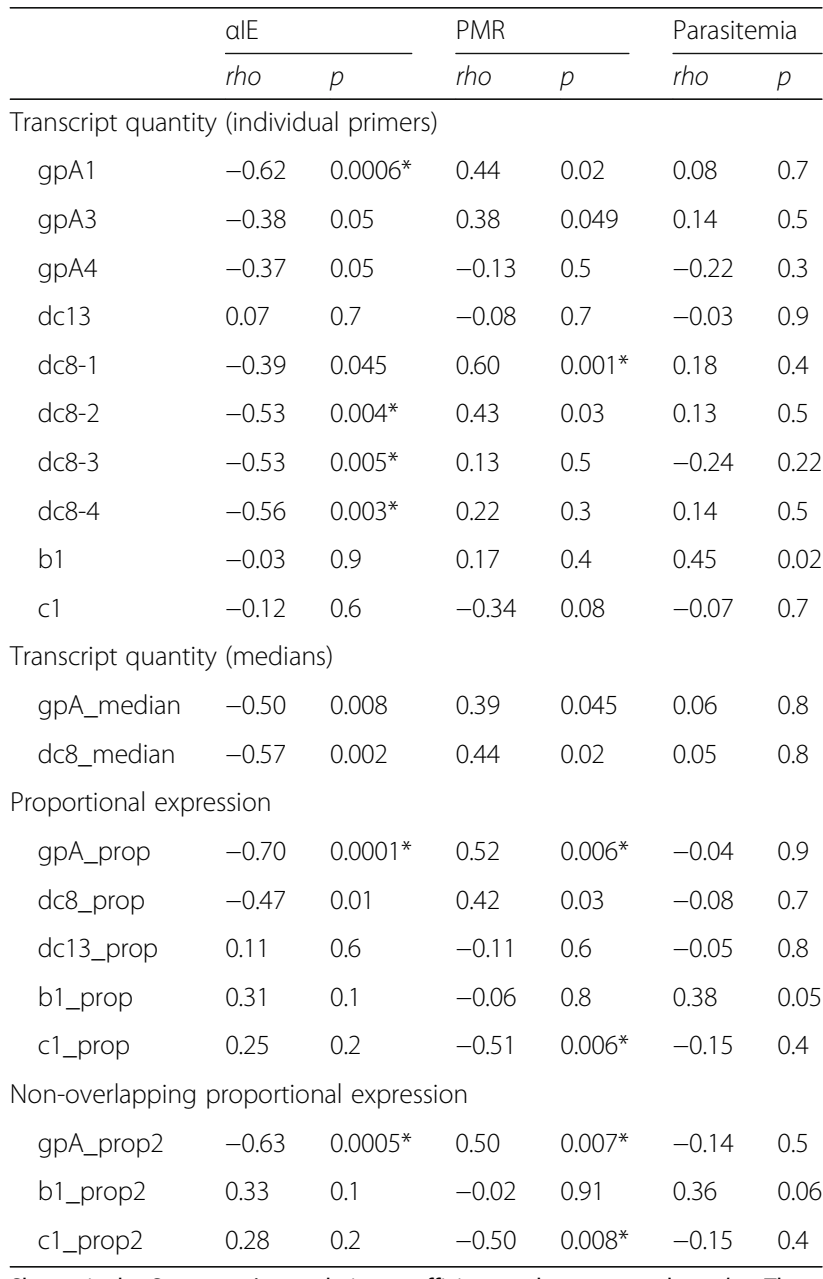

Shown is the Spearman's correlation coefficient and uncorrected $p$-value. The names of the primers listed in Table 1 were used to represent the var subclasses. *Indicate $p$-value that was significant after Bonferroni correction for multiple comparisons (10, 5 and 3 comparisons for the transcript quantity, proportional expression and non-overlapping proportional expression respectively). PMR parasite multiplication rate, aIE antibodies to IE

Table 2). The median transcript quantity of the four DC8 targeting primers (dc8_median) was also negatively associated with $\alpha$ IE (Table 2 ).

Group B and group C (as measured with the primers b1 and c1 respectively) showed no evidence for an association with $\alpha$ IE (Table 2).

In the above analyses, transcript quantities of the var subclasses were calculated relative to the average expression of two metabolic genes. This approach does not give information on the proportion contributed by each var subclass to the overall var expression of the parasite population causing the infection. To refine the analysis and better describe the relative ability of parasites expressing different PfEMP1 types to survive within infecting parasite population, we therefore estimated the 
expression of each of the broad classes of the var genes as proportion of the total measured var transcript as previously described $[40,41]$ (see methods) and assessed the relationship between the "proportional expression" values of each of the var subclass and $\alpha$ IE.

Proportional expression of Group A (gpA_prop) and DC8 (dc8_prop) showed a significant negative association with $\alpha \mathrm{IE}$ (Table 2 ) but only gpA_prop remained significant after adjusting for five comparisons (adjusted $p=0.0005$, Table 2). The associations between group $B$ and $C$ proportional expression (b1_prop and c1_prop), and $\alpha I E$ tended to be positive though not significant (Table 2).

Since different defined subgroups of var genes frequently carry shared sequence features, the transcript obtained with primer sets used to quantify the different var subclasses may overlap [40]. For examples, DC8 primers such as dc8-1 can amplify genes amplified by both the group A primers and primer b1 while the DC13 primer may amplify genes targeted by the group A primers since DC13 are a subset of group A. To estimate proportional expression of non-overlapping var classes, we excluded DC8 \& DC13 from the calculation of total transcript and recalculated the proportions. With this analysis group A proportional expression (gpA_prop2) was negatively associated with $\alpha \mathrm{IE}$ (Table 2) and remained significant after correcting for three comparisons (corrected $p=0.03)$.

var expression patterns exhibit differential associations with apparent within-host parasite multiplication rate

One of the reasons proposed to explain why group A and DC8 PfEMP1 are relatively conserved is that they are adapted to mediate high levels of cytoadhesion $[19,21,22,46]$ potentially giving parasites expressing these variants a growth advantage, by lowering the rate at which they are cleared from the circulation by the spleen. We therefore tested the relationship between the transcript quantity of the var subclasses and PMR using Spearman's rank correlation coefficient. Var transcript quantities measured using individual primer sets amplifying group A (gpA1, gpA3) and DC8 (dc8-1, dc8-2) showed a positive trend in relation to PMR (Table 2). However, only dc8-1 remained significantly associated with PMR after Bonferroni correction for 10 comparisons (corrected $p=0.01$, Table 2). In contrast, no significant correlation was seen between the transcript quantities of dc13, group B (b1) and group C (c1) and PMR (Table 2). The median transcript quantity of group A (gpA_median) and DC8 (dc8_median) were also weakly associated with PMR (Table 2).

As a secondary analysis, we again re-calculated these associations using proportional expression measures.
Like the transcript quantity, group A and DC8 proportional expressions (gpA_prop and dc8_prop) were positively associated with PMR (Table 2). In contrast, the proportional expression of group C (c1_prop) was negatively associated with PMR (Table 2). The proportional expression of DC13 and group B (dc13_prop and b1_prop) were not associated with PMR (Table 2). The association of both gpA_prop and c1_prop with PMR remained significant after adjusting for five comparisons (adjusted $p=0.03$ for both gpA_prop and c1_prop, Table 2).

Similarly, after adjusting for possible overlap between primers in this calculation, PMR was positively and negatively associated with gpA_prop2 and c1_prop2 respectively (Table 2). These associations remained significant after correcting for three comparisons (adjusted $p=0.035 \& 0.04$ for gpA_prop2 and c1_prop2 respectively, Table 2). b1_prop2 showed no evidence for an association with PMR (Table 2).

To exclude the possibility that the association between expression of the different var subclasses and PMR was an artefact of the parasitemia at the time of sampling, we tested the relationship between the var expression and sampling parasitemia $(\mathrm{p} / \mathrm{mL})$. Only the transcript quantity of group $\mathrm{B}$, which was not associated with PMR, showed a significant association with parasitemia at the time of sampling (Table 2).

\section{Antibodies to antigens on the surface of IE (aIE) provided an immunological correlate of low PMR}

If antibodies to $\alpha \mathrm{IE}$ play a direct role in the control of parasite multiplication by decreasing parasite survival, we would expect $\alpha \mathrm{IE}$ to provide the best immunological correlate of low PMR. We therefore made a comparison of our measurement of $\alpha \mathrm{IE}$ antibodies with previously published data available on the volunteers [29]. In the original study, participants were classified before CHMI into those with definite (DefExp) and minimal (MinExp) prior exposure to $P$. falciparum based on antibody levels to schizonts extract and MSP-2 [29]. All participants were diagnosed with malaria by blood-film apart from one DefExp participant (110) who was blood-film negative throughout follow-up. 18S qPCR targeting ribosomal RNA (18S rRNA) gene confirmed this subject was successfully infected with malaria parasites [29]. This volunteer had a reduced PMR (1.3) in comparison to the other twenty-seven volunteers (median PMR = 11.1) [29].

Analysis of levels of $\alpha \mathrm{IE}$ prior to CHMI showed a significant difference between the DefExp and MinExp groups ( $p=0.0007$, Fig. 1a, Mann-Whitney $\mathrm{U}$ test). The differences between the two groups hold even after excluding participant $110(p=0.001)$. Participant 110 reacted to the highest proportion of IE, surpassing the 


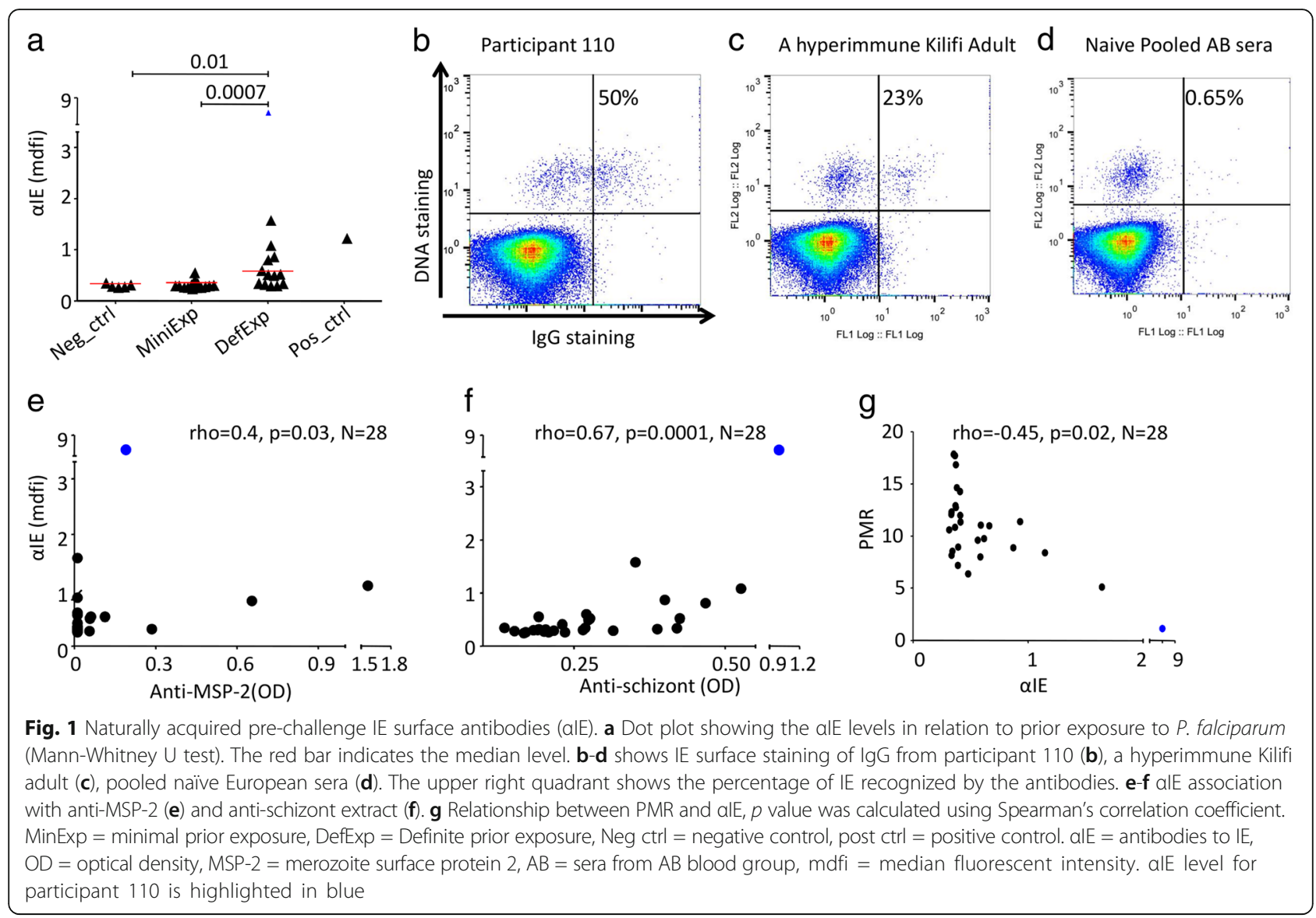

positive control $(50 \%, 23 \%$ and $0.65 \%$ for participant 110 , the positive and negative controls respectively, Fig. 1b-d)). $\alpha I E$ positively correlated with antibody levels to both MSP-2 and schizont extract (anti-MSP2 ; rho $=0.4, p=0.03$, anti-schizont; rho $=0.67$, $p=0.0001$, Fig. 1e-f).

In these volunteers, antibodies to schizonts extract were negatively associated with the PMR [29] and no associations were observed with antibodies against MSP-2 [29]. We therefore tested the relationship between the $\alpha \mathrm{IE}$ present before CHMI and the PMR. Consistent with the anti-schizont antibodies, the $\alpha \mathrm{IE}$ was negatively associated with the PMR (rho $=-0.45, p=0.01, N=28$, Fig. 1g). When we excluded participant 110 who had very high $\alpha I E$, this association remained significant (rho $=0.4, p=0.04, N=27$ ). To test how $\alpha$ IE compares to anti-schizonts extract in predicting PMR, we used two linear regression models predicting PMR using either of them as an explanatory variable. As expected, both antibodies predicted PMR ((coeff $(95 \% \mathrm{CI})$, antischizont $=1.0(0.16,1.84), p=0.02 ; \alpha \mathrm{IE}=2.60(1.20,4.0)$, $p=0.001)$. However, $\alpha$ IE explained $33 \%$ of the variations in PMR (adjusted $\mathrm{R}^{2}=33 \%$ ) while anti-schizont antibodies explained only $15.6 \%$ (adjusted $R^{2}=15.6 \%$ ), suggesting $\alpha \mathrm{IE}$ may be a better immunological correlate of
PMR. Results from linear regression were consistent with those obtained using non-parametric Spearman correlation analysis (associations with PMR excluding volunteer 110: MSP-2, rho $=-0.13, p=0.53$; anti-schizont, -0.31 , $p=0.11 ; \alpha \mathrm{IE}$, rho $=-0.39, p=0.04$ ).

Expression of a gene containing a CIDRa1.1 is associated with PMR independently of pre-challenge alE antibody levels Given that 1) both $\alpha \mathrm{IE}$ and expression of specific subclasses of var genes were associated with PMR (Fig. $1 \mathrm{~g}$ and Table 2) and 2) the expression of the subclasses of var genes were associated with the $\alpha \mathrm{IE}$ (Table 2), we sought to dissect this network of relationships further to gain insight into possible causal pathways. Specifically, we tested for evidence that specific var classes were independently associated with higher PMR. We again used linear regression models predicting PMR. All variables whose association with PMR reached an unadjusted $p<0.05$ level of significance using Spearman's rank correlation test (Table 2 and Fig. 1g) were used as explanatory variables. These explanatory variables were considered in turn in 11 separate models predicting PMR.

To determine the level of independence of $\alpha \mathrm{IE}$ and var expression in predicting PMR, we considered each 
of the var gene expression estimates in combination with $\alpha$ IE. As shown in Table 3 models 1-11, consistent with the Spearman correlation analysis, each of the variables was significantly associated with PMR when used as the sole explanatory variable. Overall, the results suggest that the associations between expression of group A and DC8 var subclasses and PMR is confounded by the level of circulating $\alpha \mathrm{IE}$ antibodies, as the expression of group A and DC8 no longer remained significantly associated with PMR after correction for prior levels of these antibodies (group A: models 12, 13, 15, 17, 20, DC8: models 16 and 18, Table 3).

Table 3 The relationship between alE antibodies before CHMI, expression of specific var subclasses after $\mathrm{CHMl}$ and PMR (outcome measure). uncorrected $p$-values are shown

\begin{tabular}{|c|c|c|c|c|}
\hline Models & $\begin{array}{l}\text { Explanatory } \\
\text { variables }\end{array}$ & Coeff(95\% Cl) & $p$-value & Adjusted $\mathrm{R}^{2}$ \\
\hline 1 & gpA1 & $1.18(0.06,2.30)$ & 0.04 & $13 \%$ \\
\hline 2 & gpA3 & $1.55(0.06,3.05)$ & 0.04 & $12 \%$ \\
\hline 3 & dc8-1 & $1.73(0.62,2.85)$ & 0.004 & $26 \%$ \\
\hline 4 & gpA_median & $0.33(0.03,0.63)$ & 0.03 & $14 \%$ \\
\hline 5 & Dc8_median & $1.32(0.07,2.58)$ & 0.04 & $12 \%$ \\
\hline 6 & gpA_prop & $7.88(2.73,13.02)$ & 0.004 & $26 \%$ \\
\hline 7 & dc8_prop & $7.85(-0.09,15.79)$ & 0.05 & $11 \%$ \\
\hline 8 & c1_prop & $-9.26(-14.85,-3.66)$ & 0.003 & $29 \%$ \\
\hline 9 & gpA_prop2 & $7.25(2.77,11.73)$ & 0.003 & $28 \%$ \\
\hline 10 & c1_prop2 & $-8.93(-14.50,-3.37)$ & 0.003 & $28 \%$ \\
\hline 11 & $\mathrm{alE}$ & $1.99(0.42,3.55)$ & 0.02 & $18 \%$ \\
\hline \multirow[t]{2}{*}{12} & gpA1 & $0.42(-1.12,1.97)$ & 0.6 & $16 \%$ \\
\hline & $\mathrm{alE}$ & $1.55(-0.69,3.80)$ & 0.17 & \\
\hline \multirow[t]{2}{*}{13} & gpA3 & $0.91(-0.69,2.52)$ & 0.3 & $20 \%$ \\
\hline & $\mathrm{alE}$ & $1.54(-0.21,3.28)$ & 0.08 & \\
\hline \multirow[t]{2}{*}{14} & dc8-1 & $1.32(0.06,2.58)$ & 0.04 & $29 \%$ \\
\hline & $\mathrm{alE}$ & $1.12(-0.56,2.80)$ & 0.2 & \\
\hline \multirow[t]{2}{*}{15} & gpA_median & $0.18(-0.18,0.53)$ & 0.3 & $19 \%$ \\
\hline & $\mathrm{alE}$ & $1.44(-0.46,3.35)$ & 0.1 & \\
\hline \multirow[t]{2}{*}{16} & dc8_median & $0.66(-0.81,2.14)$ & 0.4 & $18 \%$ \\
\hline & $\mathrm{alE}$ & $1.50(-0.40,3.41)$ & 0.1 & \\
\hline \multirow[t]{2}{*}{17} & gpA_prop & $6.0(-1.08,13.10)$ & 0.1 & $25 \%$ \\
\hline & $\mathrm{alE}$ & $0.80(-1.26,2.85)$ & 0.4 & \\
\hline \multirow[t]{2}{*}{18} & dc8_prop & $4.65(-3.67,12.96)$ & 0.3 & $19 \%$ \\
\hline & $\mathrm{alE}$ & $1.59(-0.12,3.31)$ & 0.07 & \\
\hline \multirow[t]{2}{*}{19} & c1_prop & $-7.31(-13.45,-1.17)$ & 0.02 & $32 \%$ \\
\hline & $\mathrm{alE}$ & $1.13(-0.47,2.73)$ & 0.2 & \\
\hline \multirow[t]{2}{*}{20} & gpA_prop2 & $5.80(-0.32,11.92)$ & 0.06 & $26 \%$ \\
\hline & $\mathrm{alE}$ & $0.71(-1.30,2.72)$ & 0.5 & \\
\hline \multirow[t]{2}{*}{21} & C1_prop2 & $-7.0(-13.03,-0.95)$ & 0.03 & $31 \%$ \\
\hline & $\mathrm{alE}$ & $1.18(-0.43,2.78)$ & 0.1 & \\
\hline
\end{tabular}

However, 1) the expression of DC8 var amplified with the primer dc8-1, and 2) the measure of group $\mathrm{C}$ proportional expression, showed independent associations with PMR after adjusting for $\alpha \mathrm{IE}$, positively in the case of dc8-1 and negatively in the case of group C (Table 3 model 14, 19 and 21). Primer dc8-1 is known to amplify a single gene PFD0020c in the NF54-derived parasite line 3D7 [16], and expression of this gene was not strongly associated with $\alpha \mathrm{IE}$ antibodies. This may suggest that this gene is capable of both evading host antibodies in this group of volunteers and promoting parasite survival.

\section{Discussion}

We used controlled human malaria infection of Kenyan volunteers with varying levels of naturally acquired immunity to malaria to explore how parasites adapt to host antibodies during infections, through switching between alternative copies of PfEMP1 antigens inserted into the surface of parasite infected erythrocytes.

The aim of this study was to examine the interrelationships between antibodies to parasite infected erythrocytes $(\alpha \mathrm{IE})$ carried by volunteers before experimental infection, the apparent within host multiplication rate of parasites during the subsequent infection (PMR) and the expression of specific sub-classes of parasite var genes in the surviving parasite population at the time before the infections were drug-treated. We focused on the expression of subsets of var genes previously shown to be associated with low host immunity and severe malaria. Taken together, the results show, for the first time, in a longitudinal study that parasite group A and DC8like var expression is negatively associated with levels of $\alpha \mathrm{IE}$ carried before challenge.

PfEMP1 stimulates an antibody response whose breadth develops progressively with increasing exposure to natural $P$. falciparum infections $[5,28]$. To this effect, adults that grow up in malaria-endemic regions can control parasitemia and acquire protection against clinical malaria $[47,48]$. Sera from these adults recognize many clinical isolates [49]. This ability to control parasitemia is thought to occur partly by preventing IE cytoadhesion and sequestration in the organs, making them more susceptible to removal by the spleen [50-54].

Earlier serological studies emphasized the importance of gaps in the pre-infection repertoire of protective antibodies to specific antigenic variants in explaining individual instances of clinical malaria $[55,56]$. This subsequently developed into a model of immunity in which immune responses to a subset of dominant variants conferring enhanced parasite survival (group A and DC8) potentially explain the relatively rapid development of immunity to severe malaria relative to non-severe malaria or asymptomatic infection (Fig. 2a-c). The negative 


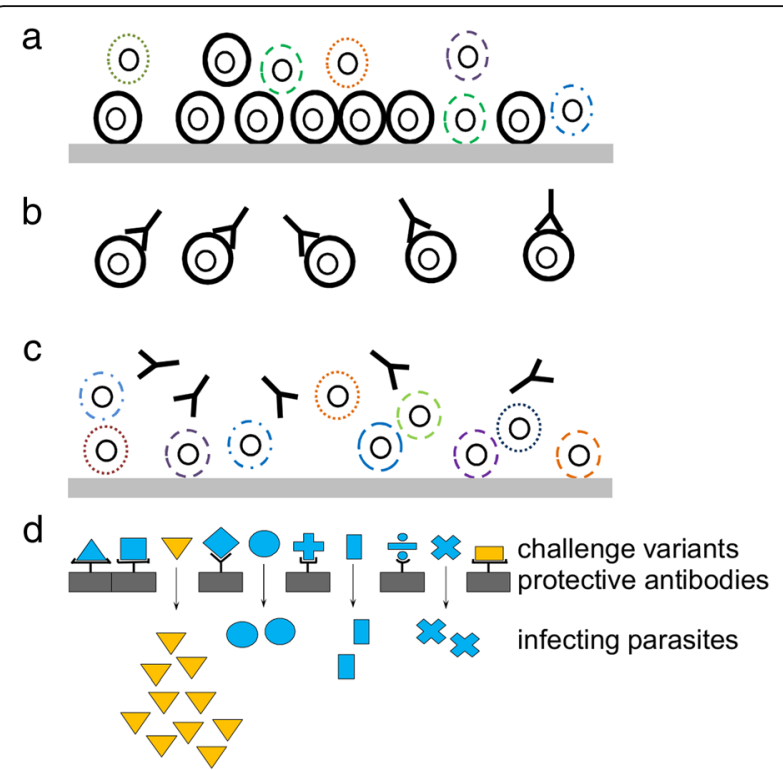

Fig. 2 Proposed model to explain the inter-relationships between antibodies, var expression and apparent parasite multiplication rate (PMR). a In the absence of alE antibodies, parasites expressing a subset of "dominant" PfEMP1 with high intrinsic cytoadhesive capacity dominate the infection (black) because of their ability to limit splenic parasite clearance rate. $\mathbf{b}$ As a result, these variants will be the first to be recognized by the developing host antibody response. $\mathbf{c}$ The surviving parasites express PfEMP1 variants that can evade antibodies, but because they have a lower intrinsic cytoadhesive capacity, these parasites have a higher splenic clearance rate, resulting in a lower observed apparent PMR. The thick grey horizontal line represents the endothelial cells that make up the inner wall of microvessels. d under a model of variant specific immunity, dominant variants (orange shapes) may arise that are poorly recognised by antibodies carried by the host population (grey rectangles) allowing them to establish infections (arrows). Their expression levels would be correlated with growth rate but poorly correlated with bulk measures of pre-infection antibodies

association between the naturally acquired $\alpha \mathrm{IE}$ and expression of group A, DC8 and PMR is consistent with a role for these antibodies against in vivo expansion of parasite population (parasite burden).

If these two general ideas are combined, we arrive at a model in which variant specific antibodies control individual members of a subset of dominant variants. Under this modified model of variant specific immunity to dominant PfEMP1, we would expect to see examples of dominant PfEMP1 that are associated with PMR, but poorly associated with prior exposure, because of low herd immunity in the host population (Fig. 2d). The single group A gene PFD0020c, predicted to be amplified by the dc8-1 primers may provide an example of such a molecule. Unless the PFD0020c identified in the NF54derived line 3D7 has undergone substantial rearrangement relative to the parent NF54 line used here, the results suggest that expression of a PFD0020c-like gene was significantly associated with PMR without a significant association with prior $\alpha \mathrm{IE}$ antibodies to local isolates. In support of this, PFD0020c from the 3D7 line was notable in previous studies. 1) unlike other group A PfEMP1, PFD0020c was poorly recognized by pooled semi immune serum [26];2) in controlled infection of naive volunteers, this gene was dominantly expressed [44]. It is perhaps also significant to note that the dbl $\beta 12$ from this PFD0020c was recently found to bind to gCq1R [57] raising the possibly of an immune modulatory effect that might conceivably reduce antibody mediated clearance. Further studies are clearly needed to quantify the expression of individual var genes in relation to domain specific antibodies against recombinant PFD0020c domains in comparison with other PfEMP1 variants from NF54 and other local parasite isolates.

This study was limited to using primers with broad specificity and relatively crude assays to assess antibody carriage before parasite challenge. However, the results demonstrate the strong potential for the exposed volunteer CHMI platform for making a detailed dissection of the host parasite interaction during the development of naturally acquired immunity. Recent studies suggest that parasites may use a similar bethedging strategy seen in the var genes to adapt to changes in their host environment [58]. Several other parasite multi-gene families (including rif and stevor encoding exported parasite antigens and others encoding proteins with diverse functions, 6-cys, clag, etramp, acs, fikk and phist a,b and c) are expressed in a clonally variant manner. Future studies using a combination of protein arrays to make fine grained measures of pre-challenge antibodies and parasite RNAseq to assess how infecting parasite populations collectively respond to antibody pressure may now provide direct insight into how parasites establish and maintain infections.

\section{Conclusions}

In summary, the results show that 1 ) naturally acquired $\alpha \mathrm{IE}$ antibodies carried before controlled infection with NF54 strain PfSPZ appear to protect preferentially against Group A, and DC8-like var gene expression 2) expression of a var gene predicted to encode a DC8-like PfEMP1 similar to 3D7 PFD0020c, was associated with higher PMR after CHMI. We propose that, in nonimmune individuals, absence or low $\alpha \mathrm{IE}$ antibodies contributes to a reduction in parasite clearance resulting in increased apparent parasite multiplication rate (PMR). Within such a model, interventions targeting specific subsets of PfEMP1 may reduce parasite growth in vivo which may in turn reduce malaria associated mortality and morbidity. 


\section{Abbreviations}

CHMl: Controlled human malaria infection; DC13: Domain cassette 13; DC8: Domain cassette 8; IE: Infected erythrocytes; PfEMP1: P. falciparum erythrocyte membrane protein 1; PfSPZ: P. falciparum Sporozoite; PMR: Parasite multiplication rate; alE: antibodies to IE

\section{Acknowledgements}

We are grateful to the study participants, the nursing, administrative and laboratory teams at the KEMRI centre for Clinical Research, Nairobi and Geographic Medicine Research, Kilifi and Sanaria's pharmaceutical operations, manufacturing, quality, regulatory, clinical and legal teams. We thank the Sanaria Inc. for providing the PfSPZ challenge used in the study.

\section{Funding}

This work was supported by the European and Developing Countries Clinical Trial Partnership (grant number: SP 2011.41304 .062 to Bernard Ogutu, Kevin Marsh, Stephen L. Hoffman, Susanne H. Hodgson); The Wellcome Trust (grant numbers: 103956 to AA, 097940 to SHH, 106917 to SJD, 084535 to PCB and KM); and The National Institute of Allergy and Infectious Disease (grant number: R44AI058375 to Stephen L. Hoffman). SJD is also a Lister Institute Research Prize Fellow and a Jenner Investigator. AA also received support from Wellcome Trust strategic grant (084538). The funders had no role in study design, data collection and analysis, decision to publish, or preparation of the manuscript.

\section{Availability of data and materials}

The dataset used in the study will be made available at https://osf.io/tdgj8/ if the manuscript is accepted.

\section{Authors' contributions}

$\mathrm{KM}, \mathrm{SHH}, \mathrm{BO}, \mathrm{SJD}$, and SLH designed the CHMI study, AA and PCB designed the current sub-study. SHH, EJ, BO, KM supervised the CHMI trial. AA and MKM generated the var expression data, CA and AA generated the alE data, DK generated the real-time PCR data used to compute PMR, FO and GK generated the ELISA data and PB contributed to the analysis of the data. AA and PCB drafted the manuscript and all authors have contributed to the writing and approved the submitted version of the manuscript.

\section{Ethics approval and consent to participate}

Ethical approval was obtained from Kenya Medical Research Institute Scientific and Ethical Review Unit (KEMRI-SERU) and written informed consent was obtained from the study participants who were all Kenyan adults. The study methods were carried out in accordance with the approved guidelines.

\section{Consent for publication}

This paper was published with the permission of the director of KEMRI.

\section{Competing interests}

Sanaria Inc. manufactured PfSPZ challenge used in the study. Thus, Stephen L. Hoffman has potential conflicts of interest.

\section{Publisher's Note}

Springer Nature remains neutral with regard to jurisdictional claims in published maps and institutional affiliations.

\section{Author details}

${ }^{1}$ KEMRI-Wellcome Trust Research Programme, CGMRC, P.O. Box 230-80108, Kilifi County, Kenya. ${ }^{2}$ Pwani University, P. O. Box 195-80108, Kilifi, Kenya. ${ }^{3}$ The Jenner Institute, University of Oxford, Oxford, UK. ${ }^{4}$ Centre for Clinical Research, Kenya Medical Research Institute, Nairobi, Kenya. ${ }^{5}$ Centre for Research in Therapeutic Sciences, Strathmore University, Nairobi, Kenya. ${ }^{6}$ Sanaria Inc., Rockville, MD, USA. ${ }^{7}$ Department of Pathology, University of Cambridge, 17 Tennis Court Road, Cambridge CB2 1QP, UK.

\section{Received: 5 August 2017 Accepted: 15 August 2017}

\section{Published online: 23 August 2017}

\section{References}

1. Gardner MJ, Hall N, Fung E, White O, Berriman M, Hyman RW, Carlton JM, Pain A, Nelson KE, Bowman S, et al. Genome sequence of the human malaria parasite plasmodium falciparum. Nature. 2002;419(6906):498-511.
2. Miller LH, Baruch DI, Marsh K, Doumbo OK. The pathogenic basis of malaria. Nature. 2002;415(6872):673-9.

3. Rowe JA, Claessens A, Corrigan RA, Arman M. Adhesion of plasmodium falciparum-infected erythrocytes to human cells: molecular mechanisms and therapeutic implications. Expert Rev Mol Med. 2009;11:e16.

4. Higgins MK, Carrington M. Sequence variation and structural conservation allows development of novel function and immune evasion in parasite surface protein families. Protein Sci. 2014;23(4):354-65.

5. Chan JA, Howell KB, Reiling L, Ataide R, Mackintosh CL, Fowkes FJ, Petter M, Chesson JM, Langer C, Warimwe GM, et al. Targets of antibodies against plasmodium falciparum-infected erythrocytes in malaria immunity. J Clin Invest. 2012;122(9):3227-38.

6. Guizetti J, Scherf A. Silence, activate, poise and switch! Mechanisms of antigenic variation in plasmodium falciparum. Cell Microbiol. 2013;15(5):718-26.

7. Rask TS, Hansen DA, Theander TG, Gorm Pedersen A, Lavstsen T. Plasmodium falciparum erythrocyte membrane protein 1 diversity in seven genomes-divide and conquer. PLoS Comput Biol. 2010;6(9):e1000933. doi:10.1371/journal.pcbi.1000933.

8. Claessens A, Hamilton WL, Kekre M, Otto TD, Faizullabhoy A, Rayner JC, Kwiatkowski D. Generation of antigenic diversity in plasmodium falciparum by structured rearrangement of Var genes during mitosis. PLoS Genet. 2014; 10(12):e1004812.

9. Bull PC, Abdi Al. The role of PfEMP1 as targets of naturally acquired immunity to childhood malaria: prospects for a vaccine. Parasitology. 2016;143(2):171-86.

10. Warimwe GM, Keane TM, Fegan G, Musyoki JN, Newton CR, Pain A, Berriman M, Marsh K, Bull PC. Plasmodium falciparum var gene expression is modified by host immunity. Proc Natl Acad Sci U S A. 2009;106(51):21801-6.

11. Warimwe GM, Fegan G, Musyoki JN, Newton CR, Opiyo M, Githinji G, Andisi C, Menza F, Kitsao B, Marsh K, et al. Prognostic indicators of life-threatening malaria are associated with distinct parasite variant antigen profiles. Sci Transl Med. 2012;4(129):129ra145.

12. Mkumbaye SI, Wang CW, Lyimo E, Jespersen JS, Manjurano A, Mosha J, Kavishe RA, Mwakalinga SB, Minja DT, Lusingu JP et al: The Severity of Plasmodium falciparum Infection Is Associated with Transcript Levels of var Genes Encoding Endothelial Protein C Receptor-Binding P. falciparum Erythrocyte Membrane Protein 1. Infect Immun 2017, 85(4).

13. Jespersen JS, Wang CW, Mkumbaye SI, Minja DT, Petersen B, Turner L, Petersen JE, Lusingu JP, Theander TG, Lavstsen T. Plasmodium falciparum var genes expressed in children with severe malaria encode CIDRalpha1 domains. EMBO Mol Med. 2016;8(8):839-50.

14. Bernabeu M, Danziger SA, Avril M, Vaz M, Babar PH, Brazier AJ, Herricks T, Maki JN, Pereira L, Mascarenhas A, et al. Severe adult malaria is associated with specific PfEMP1 adhesion types and high parasite biomass. Proc Natl Acad Sci U S A. 2016;113(23):E3270-9.

15. Bertin Gl, Lavstsen T, Guillonneau F, Doritchamou J, Wang CW, Jespersen JS, Ezimegnon S, Fievet N, Alao MJ, Lalya F, et al. Expression of the domain cassette 8 plasmodium falciparum erythrocyte membrane protein 1 is associated with cerebral malaria in Benin. PLoS One. 2013;8(7):e68368.

16. Lavstsen T, Turner L, Saguti F, Magistrado P, Rask TS, Jespersen JS, Wang CW, Berger SS, Baraka V, Marquard AM, et al. Plasmodium falciparum erythrocyte membrane protein 1 domain cassettes 8 and 13 are associated with severe malaria in children. Proc Natl Acad Sci U S A. 2012;109(26):E1791-800.

17. Kyriacou HM, Stone GN, Challis RJ, Raza A, Lyke KE, Thera MA, Kone AK, Doumbo OK, Plowe CV, Rowe JA. Differential var gene transcription in plasmodium falciparum isolates from patients with cerebral malaria compared to hyperparasitaemia. Mol Biochem Parasitol. 2006;150(2):211-8.

18. Normark J, Nilsson D, Ribacke U, Winter G, Moll K, Wheelock CE, Bayarugaba J, Kironde F, Egwang TG, Chen Q, et al. PfEMP1-DBL1alpha amino acid motifs in severe disease states of plasmodium falciparum malaria. Proc Natl Acad Sci U S A. 2007;104(40):15835-40.

19. Turner L, Lavstsen T, Berger SS, Wang CW, Petersen JE, Avril M, Brazier AJ, Freeth $J$, Jespersen JS, Nielsen MA, et al. Severe malaria is associated with parasite binding to endothelial protein C receptor. Nature. 2013;498(7455):502-5.

20. Avril M, Brazier AJ, Melcher M, Sampath S, Smith JD. DC8 and DC13 var genes associated with severe malaria bind avidly to diverse endothelial cells. PLoS Pathog. 2013;9(6):e1003430.

21. Avril M, Tripathi AK, Brazier AJ, Andisi C, Janes JH, Soma VL, Sullivan DJ Jr, Bull PC, Stins MF, Smith JD. A restricted subset of var genes mediates adherence of plasmodium falciparum-infected erythrocytes to brain endothelial cells. Proc Natl Acad Sci U S A. 2012;109(26):E1782-90. 
22. Claessens A, Adams Y, Ghumra A, Lindergard G, Buchan CC, Andisi C, Bull PC, Mok S, Gupta AP, Wang CW, et al. A subset of group A-like var genes encodes the malaria parasite ligands for binding to human brain endothelial cells. Proc Natl Acad Sci U S A. 2012;109(26):E1772-81.

23. Bull PC, Kortok M, Kai O, Ndungu F, Ross A, Lowe BS, Newbold Cl, Marsh K. Plasmodium falciparum-infected erythrocytes: agglutination by diverse Kenyan plasma is associated with severe disease and young host age. J Infect Dis. 2000;182(1):252-9.

24. Nielsen MA, Staalsoe T, Kurtzhals JA, Goka BQ, Dodoo D, Alifrangis M, Theander TG, Akanmori BD, Hviid L. Plasmodium falciparum variant surface antigen expression varies between isolates causing severe and nonsevere malaria and is modified by acquired immunity. J Immunol. 2002;168(7):3444-50.

25. Staalsoe T, Nielsen MA, Vestergaard LS, Jensen AT, Theander TG, Hviid L. In vitro selection of plasmodium falciparum 3D7 for expression of variant surface antigens associated with severe malaria in African children. Parasite Immunol. 2003;25(8-9):421-7.

26. Jensen AT, Magistrado P, Sharp S, Joergensen L, Lavstsen T, Chiucchiuini A Salanti A, Vestergaard LS, Lusingu JP, Hermsen R, et al. Plasmodium falciparum associated with severe childhood malaria preferentially expresses PfEMP1 encoded by group a var genes. J Exp Med. 2004;199(9):1179-90.

27. Cham GK, Turner L, Lusingu J, Vestergaard L, Mmbando BP, Kurtis JD, Jensen AT, Salanti A, Lavstsen T, Theander TG. Sequential, ordered acquisition of antibodies to plasmodium falciparum erythrocyte membrane protein 1 domains. J Immunol. 2009;183(5):3356-63.

28. Warimwe GM, Abdi Al, Muthui M, Fegan G, Musyoki JN, Marsh K, Bull PC Serological conservation of parasite-infected erythrocytes predicts plasmodium falciparum erythrocyte membrane protein 1 gene expression but not severity of childhood malaria. Infect Immun. 2016;84(5):1331-5.

29. Hodgson SH, Juma E, Salim A, Magiri C, Kimani D, Njenga D, Muia A, Cole $\mathrm{AO}$, Ogwang $\mathrm{C}$, Awuondo $\mathrm{K}$, et al. Evaluating controlled human malaria infection in Kenyan adults with varying degrees of prior exposure to plasmodium falciparum using sporozoites administered by intramuscular injection. Front Microbiol. 2014;5:686.

30. Epstein JE. Taking a bite out of malaria: controlled human malaria infection by needle and syringe. Am J Trop Med Hyg. 2013;88(1):3-4.

31. Osier FH, Fegan G, Polley SD, Murungi L, Verra F, Tetteh KK, Lowe B, Mwangi T, Bull PC, Thomas AW, et al. Breadth and magnitude of antibody responses to multiple plasmodium falciparum merozoite antigens are associated with protection from clinical malaria. Infect Immun. 2008;76(5):2240-8.

32. McCallum FJ, Persson KE, Mugyenyi CK, Fowkes FJ, Simpson JA, Richards JS, Williams TN, Marsh K, Beeson JG. Acquisition of growth-inhibitory antibodies against blood-stage plasmodium falciparum. PLoS One. 2008;3(10):e3571.

33. Polley SD, Conway DJ, Cavanagh DR, McBride JS, Lowe BS, Williams TN, Mwangi TW, Marsh K. High levels of serum antibodies to merozoite surface protein 2 of plasmodium falciparum are associated with reduced risk of clinical malaria in coastal Kenya. Vaccine. 2006;24(19):4233-46.

34. Sheehy SH, Duncan CJ, Elias SC, Choudhary P, Biswas S, Halstead FD, Collins KA, Edwards NJ, Douglas AD, Anagnostou NA, et al. ChAd63-MVA-vectored bloodstage malaria vaccines targeting MSP1 and AMA1: assessment of efficacy against mosquito bite challenge in humans. Mol Ther. 2012;20(12):2355-68.

35. White NJ, Pukrittayakamee S, Hien TT, Faiz MA, Mokuolu OA, Dondorp AM. Malaria. Lancet. 2014;383(9918):723-35.

36. Roestenberg M, O'Hara GA, Duncan CJ, Epstein JE, Edwards NJ, Scholzen A, van der Ven AJ, Hermsen CC, Hill AV, Sauerwein RW. Comparison of clinical and parasitological data from controlled human malaria infection trials. PLoS One. 2012;7(6):e38434.

37. Payne RO, Milne KH, Elias SC, Edwards NJ, Douglas AD, Brown RE, Silk SE, Biswas S, Miura K, Roberts R, et al. Demonstration of the blood-stage plasmodium falciparum controlled human malaria infection model to assess efficacy of the P. Falciparum apical membrane antigen 1 vaccine, FMP2.1/AS01. J Infect Dis. 2016;213(11):1743-51.

38. Rottmann M, Lavstsen T, Mugasa JP, Kaestli M, Jensen AT, Muller D, Theander T, Beck HP. Differential expression of var gene groups is associated with morbidity caused by plasmodium falciparum infection in Tanzanian children. Infect Immun. 2006;74(7):3904-11.

39. Salanti A, Staalsoe T, Lavstsen T, Jensen AT, Sowa MP, Arnot DE, Hviid L, Theander TG. Selective upregulation of a single distinctly structured var gene in chondroitin sulphate A-adhering plasmodium falciparum involved in pregnancy-associated malaria. Mol Microbiol. 2003;49(1):179-91.
40. Abdi Al, Kariuki SM, Muthui MK, Kivisi CA, Fegan G, Gitau E, Newton CR, Bull PC. Differential plasmodium falciparum surface antigen expression among children with malarial retinopathy. Sci Rep. 2015;5:18034.

41. Abdi Al, Warimwe GM, Muthui MK, Kivisi CA, Kiragu EW, Fegan GW, Bull PC. Global selection of plasmodium falciparum virulence antigen expression by host antibodies. Sci Rep. 2016;6:19882.

42. Tan J, Pieper K, Piccoli L, Abdi A, Foglierini M, Geiger R, Tully CM, Jarrossay D, Ndungu FM, Wambua J, et al. A LAIR1 insertion generates broadly reactive antibodies against malaria variant antigens. Nature. 2016;529(7584):105-9.

43. Merrick CJ, Dzikowski R, Imamura H, Chuang J, Deitsch K, Duraisingh MT. The effect of plasmodium falciparum Sir2a histone deacetylase on clonal and longitudinal variation in expression of the var family of virulence genes. Int J Parasitol. 2010;40(1):35-43.

44. Bachmann A, Petter M, Krumkamp R, Esen M, Held J, Scholz JA, Li T, Sim BK, Hoffman SL, Kremsner PG, et al. Mosquito passage dramatically changes var gene expression in controlled human plasmodium falciparum infections. PLoS Pathog. 2016;12(4):e1005538.

45. Merrick CJ, Huttenhower C, Buckee C, Amambua-Ngwa A, Gomez-Escobar N, Walther M, Conway DJ, Duraisingh MT. Epigenetic dysregulation of virulence gene expression in severe plasmodium falciparum malaria. J Infect Dis. 2012; 205(10):1593-600.

46. Salanti A, Dahlback M, Turner L, Nielsen MA, Barfod L, Magistrado P, Jensen AT, Lavstsen T, Ofori MF, Marsh K, et al. Evidence for the involvement of VAR2CSA in pregnancy-associated malaria. J Exp Med. 2004;200(9):1197-203.

47. Langhorne J, Ndungu FM, Sponaas AM, Marsh K. Immunity to malaria: more questions than answers. Nat Immunol. 2008;9(7):725-32.

48. Wilson DB, Garnham PC, Swellengrebel NH. A review of hyperendemic malaria. Trop Dis Bull. 1950;47(8):677-98.

49. Marsh K, Howard RJ. Antigens induced on erythrocytes by P. Falciparum: expression of diverse and conserved determinants. Science. 1986;231(4734): 150-3.

50. Beeson JG, Mann EJ, Elliott SR, Lema VM, Tadesse E, Molyneux ME, Brown $\mathrm{GV}$, Rogerson SJ. Antibodies to variant surface antigens of plasmodium falciparum-infected erythrocytes and adhesion inhibitory antibodies are associated with placental malaria and have overlapping and distinct targets. J Infect Dis. 2004;189(3):540-51.

51. Chen Q, Pettersson F, Vogt AM, Schmidt B, Ahuja S, Liljestrom P, Wahlgren M. Immunization with PfEMP1-DBL1alpha generates antibodies that disrupt rosettes and protect against the sequestration of plasmodium falciparuminfected erythrocytes. Vaccine. 2004;22(21-22):2701-12.

52. Ghumra A, Semblat JP, Ataide R, Kifude C, Adams Y, Claessens A, Anong DN Bull PC, Fennell C, Arman M, et al. Induction of strain-transcending antibodies against group a PfEMP1 surface antigens from virulent malaria parasites. PLOS Pathog. 2012;8(4):e1002665.

53. Mo M, Lee HC, Kotaka M, Niang M, Gao X, lyer JK, Lescar J, Preiser $P$. The C-terminal segment of the cysteine-rich interdomain of plasmodium falciparum erythrocyte membrane protein 1 determines CD36 binding and elicits antibodies that inhibit adhesion of parasite-infected erythrocytes. Infect Immun. 2008;76(5): 1837-47.

54. Ricke CH, Staalsoe T, Koram K, Akanmori BD, Riley EM, Theander TG, Hviid L. Plasma antibodies from malaria-exposed pregnant women recognize variant surface antigens on plasmodium falciparum-infected erythrocytes in a paritydependent manner and block parasite adhesion to chondroitin sulfate a. J Immunol. 2000;165(6):3309-16.

55. Giha HA, Staalsoe T, Dodoo D, Roper C, Satti GM, Arnot DE, Hviid L, Theander TG. Antibodies to variable plasmodium falciparum-infected erythrocyte surface antigens are associated with protection from novel malaria infections. Immunol Lett. 2000;71(2):117-26.

56. Bull PC, Lowe BS, Kortok M, Molyneux CS, Newbold CI, Marsh K. Parasite antigens on the infected red cell surface are targets for naturally acquired immunity to malaria. Nat Med. 1998;4(3):358-60.

57. Magallon-Tejada A, Machevo S, Cistero P, Lavstsen T, Aide P, Rubio M, Jimenez A, Turner L, Valmaseda A, Gupta $H$, et al. Cytoadhesion to $\mathrm{gClqR}$ through plasmodium falciparum erythrocyte membrane protein 1 in severe malaria. PLoS Pathog. 2016;12(11):e1006011.

58. Rovira-Graells N, Gupta AP, Planet E, Crowley VM, Mok S, Ribas de Pouplana L, Preiser PR, Bozdech Z, Cortes A. Transcriptional variation in the malaria parasite plasmodium falciparum. Genome Res. 2012;22(5):925-38. 